\title{
On points drawn from a circle
}

\author{
Vincent Vatter \\ Department of Mathematics \\ University of Florida \\ Gainesville, Florida USA \\ vatter@ufl.edu
}

\author{
Steve Waton \\ School of Mathematics and Statistics \\ University of St Andrews \\ St Andrews, Fife, Scotland
}

Submitted: Jan 7, 2010; Accepted: Nov 8, 2011; Published: Nov 21, 2011

Mathematics Subject Classifications: 05A15, 52C10, 68Q45

\begin{abstract}
Any finite generic set of points on the unit circle corresponds to a permutation in a canonical way. We characterise and enumerate the permutations that arise from this correspondence.
\end{abstract}

\section{Introduction}

Choose $n$ points from the unit circle - no two with the same $x$-coordinate or $y$-coordinate (we call such a set of points generic; another term is noncorectilinear) — label them 1$n$ by height, reading bottom-to-top, and record these labels reading left-to-right. This operation produces a permutation. For example, the set of points shown below on the left gives the permutation 45312, the plot of which is shown on the right.

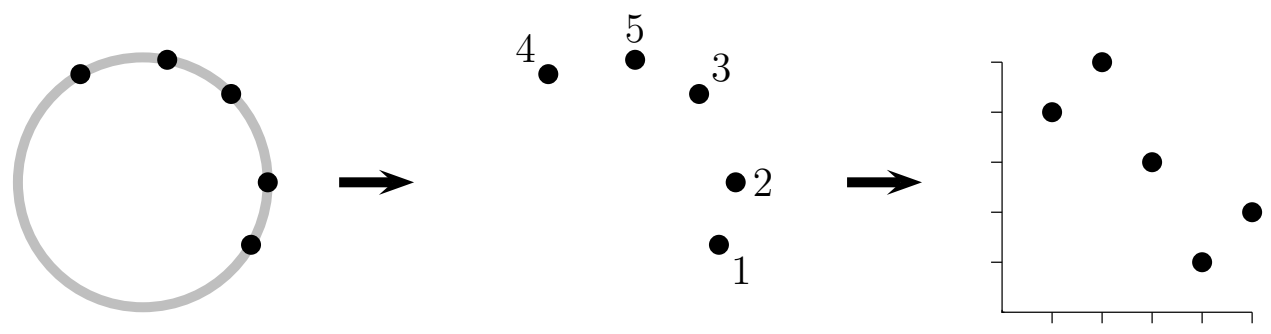

We say that such a permutation $\pi$ can be drawn on (or, from) a circle, and we refer to the points of the circle as a circle drawing of $\pi$. Not all permutations can be drawn on a circle. For example and sake of contradiction suppose that 1324 can be drawn on a circle, take such a drawing, and connect the points to give a quadrilateral such as the one 
below.

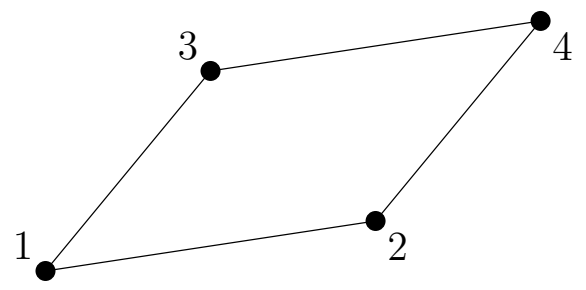

Because we have assumed that these points lie on a circle, this is a cyclic quadrilateral. However, Proposition 22 of Book III of Euclid's Elements shows that the sum of opposite angles in a cyclic quadrilateral equals $180^{\circ}$, but $\angle 213$ and $\angle 342$ must both be less than $90^{\circ}$, so 1324 (and of course its reverse, 4231) cannot be drawn on a circle.

Permutations as class representatives. In this setting, permutations function as class representatives for generic point sets in the plane under the equivalence relation of "stretching and shrinking axes". More formally, two sets $S$ and $T$ of points in the plane are said to be order isomorphic if there are strictly increasing functions $f, g: \mathbb{R} \rightarrow \mathbb{R}$ such that $\left\{\left(f\left(s_{1}\right), g\left(s_{2}\right)\right):\left(s_{1}, s_{2}\right) \in S\right\}=T$ (this is easily seen to be an equivalence relation). Every finite generic point set is order isomorphic to the plot of a unique permutation, and in practice we simply say that such a point set is order isomorphic to a permutation.

A partial order on permutations. We say that the point set $S$ is involved in the point set $T$, written $S \leq T$, if $S$ is order isomorphic to a subset of $T$. When restricted to generic point sets, this defines a partial order on permutations that can rewritten as: $\sigma$ of length $k$ is involved in $\pi$ of length $n$ if there are indices $1 \leq i_{1}<\cdots<i_{k} \leq n$ such that for all $1 \leq a, b \leq k, \sigma(a)<\sigma(b)$ if and only if $\pi\left(i_{a}\right)<\pi\left(i_{b}\right)$. In the permutation context, the involvement order is also called the pattern-containment order. Conversely, if $\sigma \not \leq \pi$ then $\pi$ is said to avoid $\sigma$. For a set $B$ of permutations, we denote by $\operatorname{Av}(B)$ the set of all permutations that avoid every element of $B$.

We refer to a downset of permutations in this involvement order (i.e., a set $\mathcal{C}$ such that whenever $\pi \in \mathcal{C}$ and $\sigma \leq \pi, \sigma \in \mathcal{C})$ as a permutation class. The set of permutations that can be drawn on a circle is easily seen to form a permutation class: if $\pi$ can be drawn on a circle and $\sigma \leq \pi$, then a circle drawing of $\sigma$ can be found inside any circle drawing of $\pi$. Thus we refer to these permutations as the circle class. Every permutation class can be expressed in the form $\operatorname{Av}(B)$ for some set $B$; indeed, $B$ may be taken to be an antichain, i.e., a set of pairwise incomparable elements. Such a set $B$ is referred to as the basis for $\mathcal{C}$.

In the next section we characterise the circle class by finding its basis. Section 3 contains the enumeration of these permutations. Finally, Section 4 considers subclasses of the circle class.

\section{Characterisation}

For the rest of the paper, we label the leftmost point of $\pi$ as $L$, the rightmost as $R$, the topmost as $T$, and the bottommost as $B$. Note that these labels can coincide. 
Proposition 2.1. If the permutation $\pi$ of length at least 2 can be drawn on a circle then there is a circle drawing of $\pi$ in which either $L$ or $R$ lies on the $x$-axis and either $T$ or $B$ lies on the $y$-axis.

Proof. Consider any circle drawing of $\pi$. We may assume by symmetry that $T$ lies closer to the $y$-axis than $B$. Note that there cannot be any points closer to the $y$-axis than $T$; if such a point lied on the lower half circle then it would be lower than $B$, while if it lied on the upper half circle it would be greater than $T$. Thus we can shift $T$ to the $y$-axis without changing order isomorphism.

We now consider this new circle drawing of $\pi$. Assume, again by symmetry, that $L$ lies closer to the $x$-axis than $R$. Note that $L \neq T$, since no point lies as far away from the $x$-axis than $T$ and $\pi$ has length at least 2 . By a similar argument as in the $T / B$ case it follows that there are no points closer to the $x$-axis than $L$, and thus we may also draw $L$ on the $x$-axis without changing order isomorphism.

Once $L$ or $T$ is placed on the $x$-axis and $T$ or $B$ is placed on the $y$-axis, the quadrants of the other points are fixed. Thus Proposition 2.1 shows that we may restrict our attention to four types of circle drawings. We refer to these (when they exist) as $L T, R T, L B$, and $R B$ drawings, based on the points that lie on the axes.

If $\pi$ can be drawn with $L$ on the $x$-axis and $a$ and $b$ are points in such a drawing, both below $L$, then $a$ is closer to the $x$-axis if and only if $a$ lies above $b$ (in either the drawing or the permutation, both are equivalent). Similarly, if $a$ and $b$ both lie above $L, a$ is closer to the $x$-axis if and only if $a$ lies below $b$. Motivated by these observations, we define the linear orders

$$
a<_{L}^{-} b \Longleftrightarrow a \text { lies above } b
$$

on $L$ and the points below $L$, and

$$
a<_{L}^{+} b \Longleftrightarrow a \text { lies below } b
$$

on $L$ and the points above $L$. The same holds with $L$ replaced by $R$, and we define $<_{R}^{-}$ and $<_{R}^{+}$analogously.

Similarly, if $\pi$ can be drawn with $T$ on the $y$-axis and $a$ and $b$ lie to the left of $T$ in such a drawing, $a$ lies closer to the $x$-axis if and only if $a$ lies to the left of $b$. We thus define

$$
a<_{T}^{-} b \Longleftrightarrow a \text { lies to the left of } b
$$

on $T$ and the points to the left of $T$, and

$$
a<_{T}^{+} b \Longleftrightarrow a \text { lies to the right of } b
$$

on $T$ and the points to the right of $T$. The orders $<_{B}^{-}$and $<_{B}^{+}$are defined by replacing $T$ by $B$.

Thus we have four linear orders on (subsets of) the points of $\pi$ for each of the four types of circle drawings we are considering. For example, the LT orders are $\left\{<_{L}^{-},<_{L}^{+},<_{T}^{-},<_{T}^{+}\right\}$. To continue this example, from an $L T$ drawing of $\pi$ in which no two points lie the same 
distance from the $x$-axis (which can always be achieved by shifting the points by $\varepsilon$ ) we can build a linear order $<_{x}$ on the points of $\pi$ by defining

$$
a<_{x} b \Longleftrightarrow a \text { lies closer to the } x \text {-axis than } b \text {. }
$$

The importance of this order is that each of the $L T$ orders agrees with $<_{x}$; i.e., if $a<b$ for some $<\in\left\{<_{L}^{-},<_{L}^{+},<_{T}^{-},<_{T}^{+}\right\}$, then $a<_{x} b$. In other words, if $\pi$ has an $L T$ drawing then the $L T$ orders are consistent: there does not exist a cycle

$$
a_{1}<_{1} a_{2}<_{2} \cdots<_{k} a_{1}
$$

where each $<_{i} \in\left\{<_{L}^{-},<_{L}^{+},<_{T}^{-},<_{T}^{+}\right\}$. Because these orders are consistent, we can define the LT poset as the transitive closure of the union of these orders, i.e., as the poset on the points of $\pi$ in which

$$
a<_{L T} b \Longleftrightarrow a=a_{1}<_{1} a_{2}<_{2} \cdots<_{k} a_{k}=b
$$

where each $<_{i} \in\left\{<_{L}^{-},<_{L}^{+},<_{T}^{-},<_{T}^{+}\right\}$. In this language, our previous comment was that $<_{x}$ is a linear extension of $<_{L T}$. Conversely, any linear extension of this poset naturally produces a circle drawing, as verified below.

Proposition 2.2. If the $L T$ (resp. RT, LB, RB) orders for $\pi$ are consistent, then $\pi$ has an $L T$ (resp. $R T, L B, R B$ ) drawing.

Proof, accompanied by Figure 1. By symmetry we may assume that the $L T$ orders for $\pi$ are consistent. Consider a linear extension $a_{1}<\cdots<a_{n}$ of the $L T$ poset of $\pi$, and note that $a_{1}$ must be $L$ and $a_{n}$ must be $T$. We begin by placing $a_{1}$ on the $x$-axis. Now set $\theta=\pi / 2(n-1)$ and for $j=2, \ldots, n-1$ place $a_{j}$ at $(\cos (j \theta), \sin (j \theta))$ if $a_{j}$ lies above $L$ and to the right of $T,(\cos (\pi / 2-j \theta), \sin (\pi / 2-j \theta))$ if $a_{j}$ lies above $L$ and to the left of $T$, $(\cos (\pi / 2+j \theta), \sin (\pi / 2+j \theta))$ if $a_{j}$ lies below $L$ to the left of $T$, and $(\cos (-j \theta), \sin (-j \theta))$ if $a_{j}$ lies below $L$ to the right of $T$. We finish by placing $a_{n}=T$ on the $y$-axis.

This proposition allows us to finish the characterisation of the circle class:

Theorem 2.3. The basis elements for the circle class are those plotted in Figure 2.

Proof. Suppose that $\pi$ cannot be drawn on a circle. Thus by Proposition 2.2, each of the four sets of linear orders are inconsistent. Suppose, e.g., that the $L T$ orders for $\pi$ are inconsistent, or in other words, that they contain a cycle, and consider a cycle of minimal length, say

$$
a_{1}<_{1} a_{2}<_{2} \cdots<_{k} a_{1},
$$

where each $<_{i} \in\left\{<_{L}^{-},<_{L}^{+},<_{T}^{-},<_{T}^{+}\right\}$. Each $a_{i}$ except $a_{1}$ must be distinct, and $a_{1}$ may occur only twice, since otherwise the cycle could be shortened. Similarly, each of $<_{L}^{-},<_{L}^{+},<_{T}^{-}$, and $<_{T}^{+}$can occur at must once in a minimal cycle, so the length of the cycle is at most 4 . 

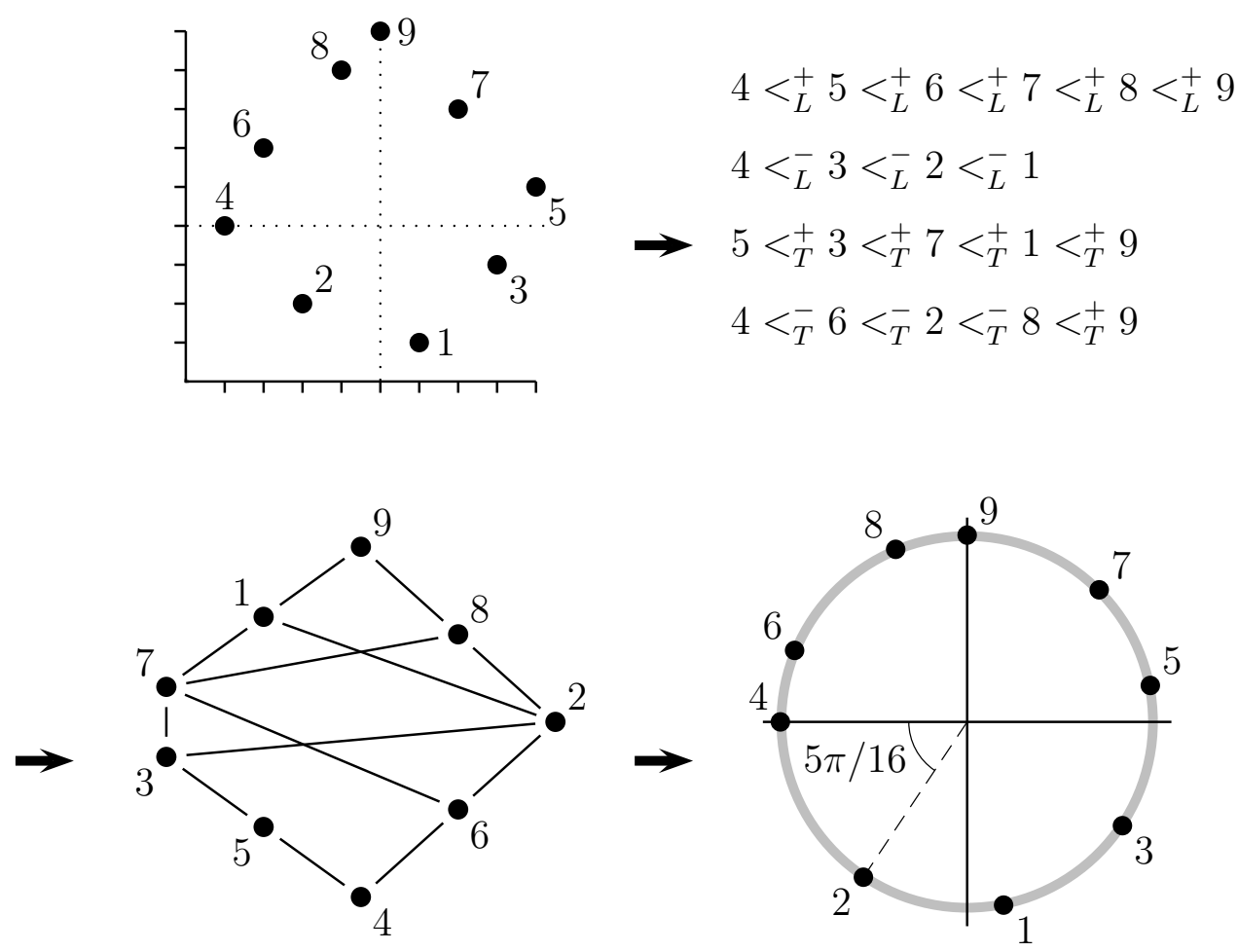

Figure 1: The construction of an $L T$ drawing of the permutation 462891735. In this case we took the linear extension $4<5<6<3<7<2<8<1<9$.
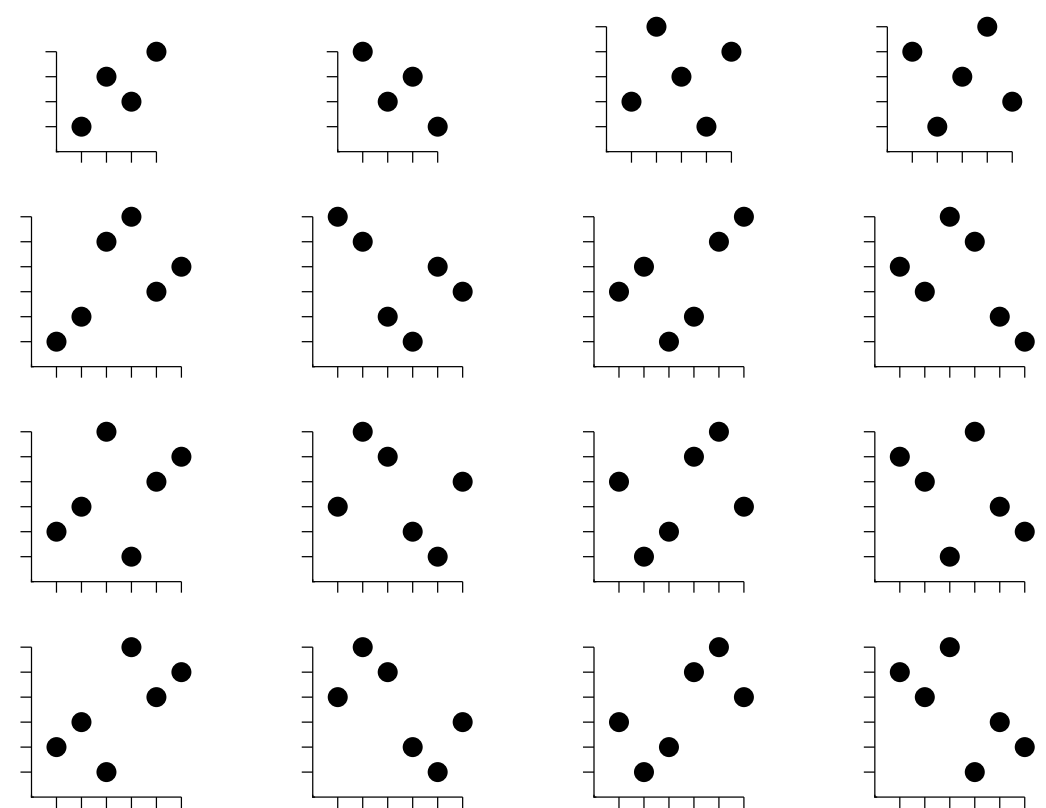

Figure 2: The basis for the class of permutations that can be drawn on a circle. 
Moreover, each point (except $L$ and $T$, but these points cannot participate in a cycle) are ordered by precisely one of $\left\{<_{L}^{-},<_{L}^{+}\right\}$and precisely one of $\left\{<_{T}^{-},<_{T}^{+}\right\}$. Thus cycles of length 3 are impossible. Now consider a minimal cycle of length 4 . There are two cases. If this cycle is of the form

$$
a_{1}<_{L}^{+} a_{2}<_{T}^{+} a_{3}<_{L}^{-} a_{4}<_{T}^{-} a_{1}
$$

then it is easy to check that these points are order isomorphic to 1324 :

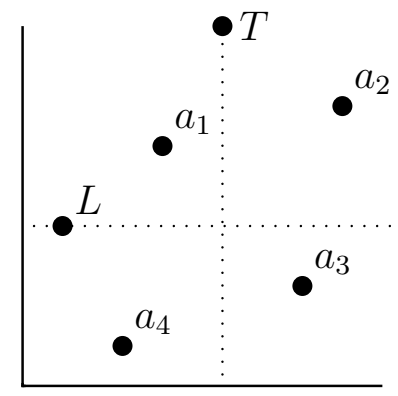

As observed in the Introduction: 1324 cannot be drawn on a circle, it is easily seen to be a basis element for this class, and is included in Figure 2. In the other case, $a_{1}>_{L}^{+} a_{2}>_{T}^{+} a_{3}>_{L}^{-} a_{4}>_{T}^{-} a_{1}$, and it is easy to check that the points are order isomorphic to 4231, which is also a basis element and is listed in Figure 2. The same situations occur if the $R T, L B$, or $R B$ orders contain cycles of length 4 , so we may assume that this does not occur.

Thus we are may assume that each of the four sets of orders contains a cycle of length 2. Choose one such cycle for each set of orders. These (at most) eight points together with $L, R, T$, and $B$ have inconsistent $L T, R T, L B$, and $R B$ orders, and thus cannot be drawn on a circle. Therefore the length of a basis element for this class is at most 12 . A routine computer check finishes the proof.

Theorem 2.3 can also be stated in the following way, more akin to Helly's Theorem: if every permutation of length 6 or less that is involved in $\pi$ can be drawn on a circle then $\pi$ can be drawn on a circle.

\section{Enumeration}

We count the permutations that can be drawn on a circle by putting them in bijection with the words of a regular language. From any circle drawing of $\pi$ in which no two points lie the same distance from the $x$-axis, we order the points according to $<_{x}$ (the "distance from the $x$-axis" order) and record which quadrant each point lies in. In doing so we take quadrant 1 as $\{(x, y): x, y \geq 0\}$, quadrant 2 as $\{(x, y): x<0, y \geq 0\}$, quadrant 3 as $\{(x, y): x \leq 0, y<0\}$, and quadrant 4 as $\{(x, y): x>0, y<0\}$. This gives a word over the alphabet $A=\{1,2,3,4\}$ that corresponds to $\pi$; see Figure 3 for an example.

As a permutation may correspond to many different words, we need to choose a distinguished word for each permutation and then characterise the language of distinguished 

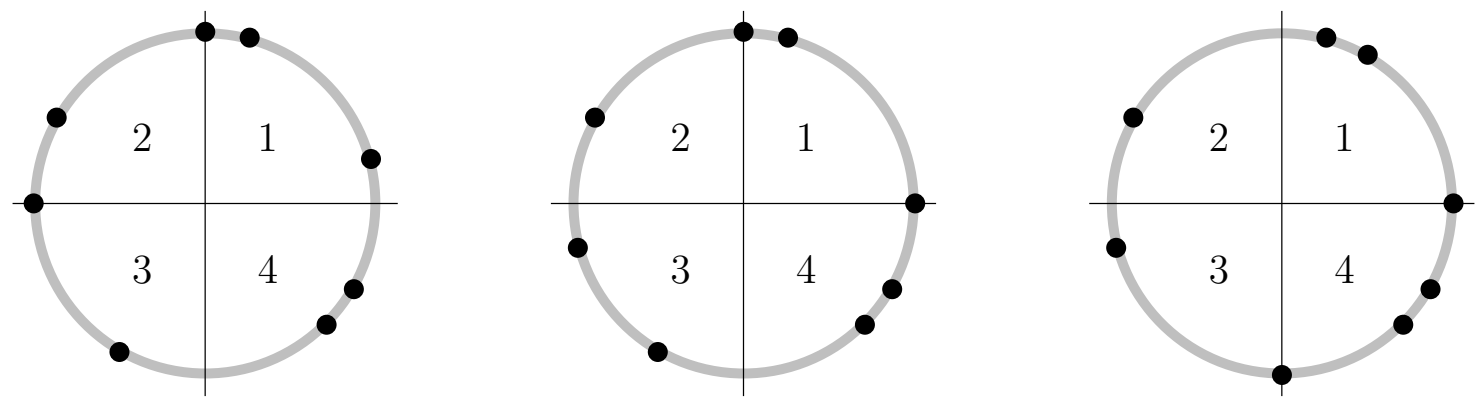

Figure 3: Three circle drawings of the permutation 46187235, giving the words 21244311, 13244311, and 13244113, from left to right. The third of these words, 13244113, happens to be the minimal word for this permutation, and corresponds to its $R B$ drawing.

words. Our choice for the distinguished word to associate to a permutation is the lexicographically minimal word (henceforth shortened to minimal word). We begin with some simple observations.

Proposition 3.1. No minimal word is of the form $3 A^{*}, 4 A^{*}, A^{*} 2$, or $A^{*} 4$.

Proof. It is follows from the proof of Proposition 2.1 and our labelling of quadrants that the words $2 u$ and $3 u$ correspond to the same permutation, as do the words $1 u$ and $4 u, u 1$ and $u 2$, and $u 3$ and $u 4$.

Because of Proposition 3.1, we need only consider words that come from $L T, R T, L B$, or $R B$ drawings. Indeed, each such word comes from a linear extension of one of these drawing posets. Our next observation yields a canonical linear extension for each poset.

Proposition 3.2. No minimal word is of the form $A^{*} 31 A^{*}$ or $A^{*} 42 A^{*}$.

Proof. The words $u 13 v$ and $u 31 v$ encode the same permutation, as do $u 24 v$ and $u 42 v$.

Proposition 3.2 eliminates all but one word that arises from each of the four posets. Put another way, every permutation that can be drawn on a circle corresponds to at most four words in the language

$$
A^{*} \backslash\left(3 A^{*} \cup 4 A^{*} \cup A^{*} 2 \cup A^{*} 4 \cup A^{*} 42 A^{*} \cup A^{*} 31\right) .
$$

We now introduce terms for the permutations that correspond to more than one word in the language above, i.e., the permutations that have more than one drawing poset.

- We say that $\pi$ has an ambiguous beginning if both $L$ and $R$ can be drawn on the $x$-axis.

- We say that $\pi$ has an ambiguous ending if both $T$ and $B$ can be drawn on the $y$-axis. 

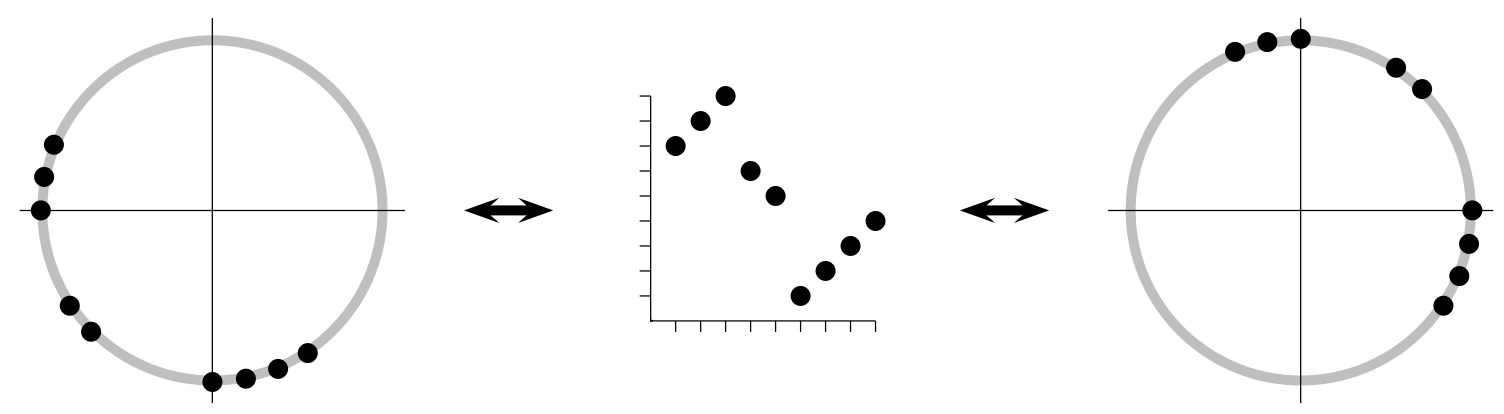

Figure 4: An epicene permutation drawn in two very different ways.

We must pay particular attention to a particular kind of ambiguity; we say a permutation is epicene if it consists of three distinct monotonic subpermutations in one of two configurations. Either an increase, above and to the left of a decrease, which is in turn above and to the left of an increase, or a decrease, below and to the left of an increase, which is in turn below and to the left of a decrease. Epicene permutations admit two radically different drawings - see Figure 4 - and so need extra consideration.

We now introduce an extension of Proposition 2.1. We state only the $L / R$ version, the $T / B$ version follows by symmetry.

Proposition 3.3. Let $\pi$ be a permutation which can be drawn on a circle with either $L$ or $R$ on the $x$-axis. There are points vertically between $L$ and $R$ if and only if $\pi$ is an epicene permutation.

Proof. Suppose that $\pi$ satisfies these hypotheses and assume (by symmetry) that $L$ lies above $R$. In any $L$ drawing of $\pi$ (i.e., any drawing with $L$ on the $x$-axis), the points lying vertically between $L$ and $R$ must lie in quadrant 3 (because they cannot lie to the right of $R$ ) and thus form a decreasing subsequence. These points must similarly lie in quadrant 1 in any $R$ drawing of $\pi$. Let us call this decreasing subsequence $D$. By considering the position of $D$ in both an $L$ and an $R$ drawing of $\pi$, it is apparent that no points may lie amongst $D$ either horizontally or vertically.

If there are any points above $L$ then they must lie in quadrant 2 in both drawings and thus form an increase - say $I_{1}$ — above and to the left of $D$; again, no points may lie amongst this increasing subsequence either horizontally or vertically. Finally, any and all points below $R$ must lie in quadrant 4 in both drawings and so must form an increasing subsequence $I_{2}$ below and to the right of $D$, and no points may lie amongst $I_{2}$. Thus $\pi$ consists of an increasing subsequence $\left(I_{1}\right.$ together with $\left.L\right)$ above and to the left of a decreasing subsequence $(D)$, which lies above and to the left of an increasing subsequence $\left(I_{2}\right.$ together with $R$ ) and is therefore epicene.

This proposition allows us to eliminate the remaining non-minimal words with the following remarks: 
- No minimal word is of the form $23^{*} 1 A^{*}$ or $2^{+} 4 A^{*}$, because these are non-minimal words for non-epicene permutations with ambiguous beginnings. The minimal words for such permutations are obtained from $R$ drawings.

- No minimal word is of the form $A^{*} 31^{+}$or $A^{*} 42^{*} 1$, because these are non-minimal words for non-epicene permutations with ambiguous endings. The minimal words for such permutations are obtained from $B$ drawings. (Although note that these words are already eliminated by Proposition 3.2.)

- No miminal word is of of the form $2^{+} 1^{*}, 2^{+} 3^{*}, 23^{*} 2^{*} 1^{+}$, or $2^{+} 3^{*} 4^{*} 3$, because these are non-minimal words for epicene permutations. The minimal words for such permutations are obtained from $R$ drawings.

From the regular language we have obtained, it is routine to enumerate the permutations that can be drawn on a circle.

Theorem 3.4. The generating function (by length) for permutations that can be drawn on a circle is

$$
\frac{1-6 x+12 x^{2}-10 x^{3}+5 x^{4}+2 x^{5}-2 x^{6}}{\left(1-4 x+2 x^{2}\right)(1-x)^{3}} .
$$

The sequence begins

$$
1,2,6,22,84,308,1090,3782,13000,44504,152102,519506,1773948,6056932 \text {. }
$$

\section{Subclasses and a Superclass}

While we only used the words of Section 3 for enumeration, they are also useful for describing the involvement order on permutations that can be drawn on a circle:

Proposition 4.1. Suppose $\sigma$ and $\pi$ can both be drawn on a circle. Then $\sigma \leq \pi$ if and only if every word corresponding to $\pi$ contains some word corresponding to $\sigma$.

Proof. If $\sigma \leq \pi$ then in the word that corresponds to any drawing of $\pi$, the subword given by a subset order isomorphic to $\sigma$ is equal to a word corresponding to $\sigma$, because that subset is a circle drawing of $\sigma$. Conversely, if a word corresponding to $\pi$ contains a subword that corresponds to $\sigma$, then the points given by this subword are clearly order isomorphic to $\sigma$ and thus $\sigma \leq \pi$.

As one consequence, because Higman's Theorem [3] states that there is no infinite antichain of words under the subword order, the circle class has no infinite antichain, or in other words, the circle class is partially well-ordered.

Now consider a subclass, say $\mathcal{C}$, of the circle class. This subclass can be described by the set of minimal permutations that can be drawn on a circle but do not lie in $\mathcal{C}$. Let us denote this set by $B$, with the caveat that it is not the basis for $\mathcal{C}$ but rather the "basis for $\mathcal{C}$ within the circle class". Proposition 4.1 implies that the minimal words of 
permutations in $\mathcal{C}$ are precisely the minimal words of permutations that can be drawn on a circle that do not contain as a subword any word that corresponds to an element of $B$. Because $B$ is an antichain, Higman's Theorem shows that it is finite, and clearly each permutation can correspond to only finitely many words. Therefore the set of minimal words of permutations in $\mathcal{C}$ consists of the set of minimal words from Section 3 that avoid a finite set of subwords, and thus the language they constitute is regular and has a rational generating function. We collect these observations below.

Corollary 4.2. The circle class is partially well-ordered and every subclass of the circle class has a rational generating function.

In closing we draw the reader's attention to two other permutation classes defined by pictures: the class consisting of all permutations that can be drawn in convex position (which therefore contains the circle class) is studied in Albert et al. [1], while the class of permutations that can be drawn on an $\mathbf{X}$ is studied in Elizalde [2].

Acknowledgements. We would like to thank Michael Albert and Nik Ruškuc for interesting discussions, and Julian West for his careful checking of the manuscript and many suggestions which improved the presentation. These results are drawn from the second author's $\mathrm{PhD}$ thesis [4].

\section{References}

[1] Albert, M. H., Linton, S., Ruškuc, N., Vatter, V., And Waton, S. On convex permutations. Discrete Math. 311 (2011), 715-722.

[2] Elizalde, S. The $\mathcal{X}$-class and almost-increasing permutations. Ann. Comb. 15 (2011), 51-68.

[3] Higman, G. Ordering by divisibility in abstract algebras. Proc. London Math. Soc. (3) 2 (1952), 326-336.

[4] Waton, S. On Permutation Classes Defined by Token Passing Networks, Gridding Matrices and Pictures: Three Flavours of Involvement. PhD thesis, Univ. of St Andrews, 2007. 